\title{
Three-dimensional optical manipulation of a single electron spin
}

\author{
Michael Geiselmann', Mathieu L. Juan', Jan Renger', Jana M. Say², Louise J. Brown², \\ F. Javier García de Abajo $^{3}$, Frank Koppens ${ }^{1}$ and Romain Quidant ${ }^{1,4 \star}$
}

\begin{abstract}
Nitrogen vacancy (NV) centres in diamond are promising elemental blocks for quantum optics ${ }^{1,2}$, spin-based quantum information processing ${ }^{3,4}$ and high-resolution sensing ${ }^{5-11}$. However, fully exploiting the capabilities of these NV centres requires suitable strategies to accurately manipulate them. Here, we use optical tweezers ${ }^{12}$ as a tool to achieve deterministic trapping and three-dimensional spatial manipulation of individual nanodiamonds hosting a single NV spin. Remarkably, we find that the NV axis is nearly fixed inside the trap and can be controlled in situ by adjusting the polarization of the trapping light. By combining this unique spatial and angular control with coherent manipulation of the NV spin and fluorescence lifetime measurements near an integrated photonic system, we demonstrate individual optically trapped $N V$ centres as a novel route for both three-dimensional vectorial magnetometry and sensing of the local density of optical states.
\end{abstract}

$\mathrm{NV}$ centres arise randomly from the presence of natural nitrogen impurities in diamond, but they can be created artificially at predefined positions through ion implantation on ultrapure diamond bulk crystals ${ }^{13,14}$. Alternatively, nanocrystal diamonds with sizes ranging from $\sim 5 \mathrm{~nm}$ to $100 \mathrm{~nm}$ can also host single NV centres (ref. 15). Their nanosize is advantageous because they can be transported to a location of interest, allowing one to place a single spin in very close proximity to photonic ${ }^{16,17}$ and plasmonic $^{18-23}$ systems to produce more customizable single-photon sources ${ }^{19,23}$. Furthermore, a mobile single NV centre can act as a nanoprobe of a magnetic field ${ }^{5-10}$, an electric field ${ }^{11}$ and also the electromagnetic local density of optical states (LDOS) $)^{19,24}$. Manipulation of single $\mathrm{NV}$ centres has so far been achieved by using scanning probe-based approaches such as atomic force microscopy (AFM) $6,7,9,10,21,25$. Although these approaches have led to a remarkable level of control, experimental implementation is limited to quasi-twodimensional manipulation and requires nanoscale positioning of the nanocrystal diamond on the AFM tip. Also, the presence of the host tip can affect the intrinsic properties of the NV centre as well as the local fields being probed. Furthermore, this AFM-based approach typically operates in gas environments, whereas liquidphase environments are generally required for bio-applications. Importantly, no technique has so far demonstrated in situ control over the orientation of the NV axis, which is critical for full vectorial magnetic and optical sensing based on a single spin or optical dipole, and potentially useful for the implementation of quantum information-processing schemes.

In this Letter, we experimentally demonstrate an approach based on three-dimensional optical manipulation of an individual nanocrystal diamond hosting a single NV centre. Although a similar approach led to electron-spin-resonance (ESR) measurements on a large ensemble of nanocrystal diamonds containing multiple $\mathrm{NV}$ centres ${ }^{26}$, here we actively select a single nanocrystal diamond that is diffusing in liquid. Once trapped, a high level of control of the spin position of the single NV centre is achieved along the three spatial axes with nanoscale resolution. Remarkably, the orientation of the trapped NV remains nearly fixed over time and can be accurately controlled, which allows us to implement coherent manipulation of a single $\mathrm{NV}$ spin and vectorial magnetometry. Finally, we show that lifetime mapping while raster scanning the trapped $\mathrm{NV}$ above a patterned surface provides direct insights into the electromagnetic LDOS near an integrated channel waveguide.

The nanocrystal diamonds used in our experiments had diameters of $\sim 60-70 \mathrm{~nm}$ and were dispersed in a 5:1 glycerol/water mix (refractive index $n=1.46$ ) confined in a static fluidic chamber. The optical trap was formed by tightly focusing $(\mathrm{NA}=1.2)$ to the same point two equally intense and collinearly polarized counterpropagating beams $(\sim 45 \mathrm{~mW}$ per beam $)$ from a Nd:YAG $(1,064 \mathrm{~nm}$ ) laser (Fig. 1). The stability of the trap was enhanced by cancelling out the scattering forces and producing constructive interference along the optical axis. This strategy enabled us to trap and manipulate a single nanocrystal diamond $(n=2.391)$ as small as $50 \mathrm{~nm}$ in all three spatial directions. In our experiment, optical manipulation of the nanocrystal diamond was combined with full characterization of its NV centre. At each spatial position, we performed time-correlated single-photon counting, extracted fluorescence lifetimes and performed optically detected ESR spectroscopy (see Methods) ${ }^{27,28}$.

To deterministically trap an individual nanocrystal diamond containing a single NV centre, we used a highly sensitive electronmultiplying charged coupled device (EM-CCD) camera in fluorescence imaging mode to follow the diffusion of the nanocrystal diamonds in real time (Figs 1d,e and 2). We immobilized the fluorescent nanocrystal diamond of interest by bringing the focus of the dual-beam trap close to it. To verify that this nanocrystal diamond only hosted a single NV centre, we performed coincidence measurements using Hanbury-Brown and Twiss (HBT) detection. Figure 1a shows measured antibunching below the threshold of 0.5 , proving that the trapped nanocrystal diamond contains a single NV centre. To assess the accuracy of our manipulation approach, we resolved the degree of spatial confinement of the nanocrystal diamond within the trap (see Methods) and found the full-width at half-maximum (FWHM) of the Gaussian distribution to be $80 \mathrm{~nm}$ along both transverse directions (Fig. 2, insets). From our simulations we expect its confinement in the $z$-direction (along the beams) to be smaller than $50 \mathrm{~nm}$ because of the interference fringes produced by the counter-propagating beams

${ }^{1}$ ICFO - Institut de Ciencies Fotoniques, Mediterranean Technology Park, 08860 Castelldefels (Barcelona), Spain, ${ }^{2}$ Department of Chemistry and Biomolecular Sciences, Macquarie University, Sydney, New South Wales 2109, Australia, ${ }^{3}$ IQFR - CSIC, Serrano 119, 28006 Madrid, Spain, ${ }^{4}$ ICREA - Institució Catalana de Recerca i Estudis Avançats, Barcelona, Spain. ^e-mail: romain.quidant@icfo.es 

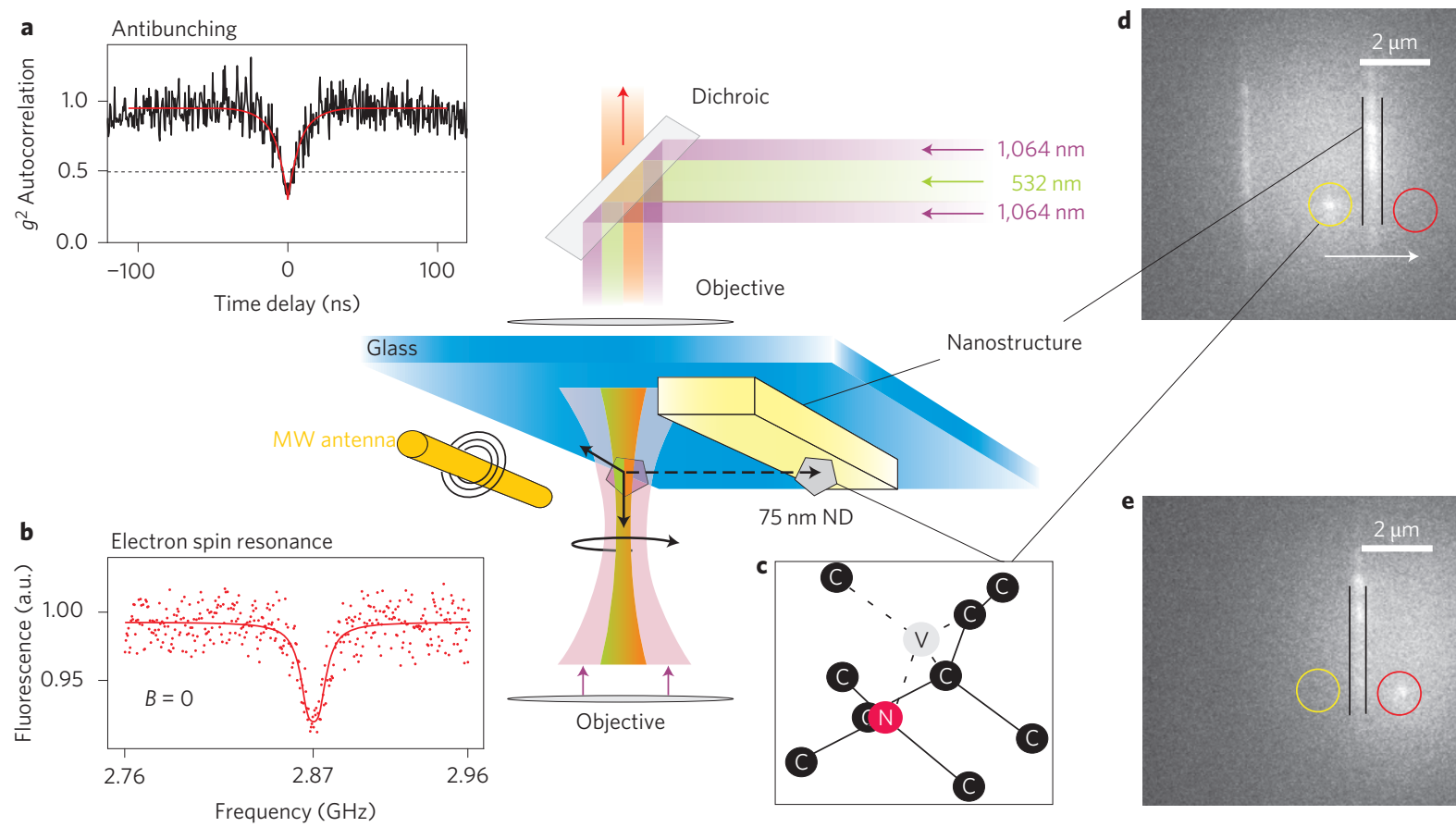

Figure 1 | Three-dimensional optical trapping and manipulation of a single electron spin. A near-infrared laser $(\lambda=1,064 \mathrm{~nm}$, shown in light violet in the central sketch) is focused through two high-NA objectives to trap a nanocrystal diamond (ND) containing a single NV centre. A superimposed $532 \mathrm{~nm}$ laser (shown in green) excites the NV. The emitted photoluminescence (orange) passes a dichroic filter and is detected by two APDs. The central schematic shows the basic elements of our experiment. a, Coincidence measurements of a trapped ND confirming the single-photon character of the NV luminescence $\left(g^{(2)}(0)<1 / 2\right)$. The red line is an exponential fit with a lifetime decay of $18 \mathrm{~ns}$. b. Optically detected electron spin resonance (ESR) of a single NV centre. c) Schematic of the NV centre in diamond consisting of a substitutional nitrogen atom (N) adjacent to a vacant lattice site (V) within the carbon (C) lattice. d,e, Scanning of a single trapped NV centre across a 250-nm-wide $\mathrm{TiO}_{2}$ waveguide (from the yellow to the red circle). The fluorescence image is taken with an EM-CCD camera while illuminating a large area with a $532 \mathrm{~nm}$ laser. The bright spot is the photoluminescence from the NV centre, and the vertical bright lines correspond to fluorescence from the $\mathrm{TiO}_{2}$ waveguide.

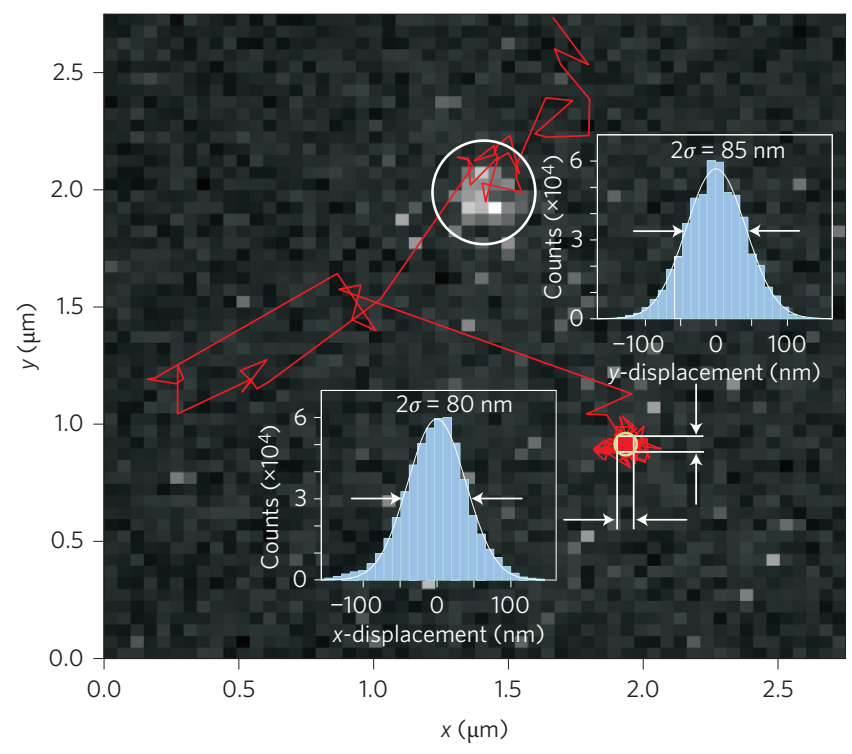

Figure 2 | Tracking and trapping of a single nanocrystal diamond with a single NV centre. EM-CCD image of a fluorescent nanodiamond (white circle) and its position trace (red line). Tracking the fluorescence makes it possible to direct the nanodiamond with the xyz piezoscanner close to the trap, where it is captured and confined. Insets: position histograms of the time trace of the trapped nanocrystal diamond retrieved from position tracking with a quadrant photodiode (QPD) ${ }^{29}$. The solid lines are Gaussian fits to the position histograms. The standard deviation is $\sim 40 \mathrm{~nm}$. The particle is confined within less than $80 \mathrm{~nm}$ for $70 \%$ of the time.
(Supplementary Fig. S11). The trap stiffness achieved is such that the nanocrystal diamond can be moved in the chamber over distances as large as $100 \mu \mathrm{m}$ with velocities up to $10 \mu \mathrm{m} \mathrm{s}^{-1}$.

This demonstration of single NV centre trapping enables us to perform vectorial magnetometry based on a single electron spin. A crucial requirement for this is the stability of the NV axis (that is, the spin quantization axis) inside the trap, which we assessed by means of optically detected ESR measurements (Fig. 3). To this end, we positioned the nanocrystal diamond in the vicinity of a gold wire microwave antenna, which was integrated into the chamber and connected to a microwave generator. The ESR spectrum was obtained by monitoring the NV fluorescence while scanning the microwave frequency over the $m_{\mathrm{s}}=0 \leftrightarrow m_{\mathrm{s}}= \pm 1$ transition at $2.87 \mathrm{GHz}$. Without any external magnetic field, the $m_{\mathrm{s}}= \pm 1$ states are degenerate, leading to a single dip in the ESR spectrum, as shown in Fig. 3a. In contrast, when we apply an external magnetic field, its vector projection along the intrinsic magnetic dipole of the NV induces Zeeman splitting of the $m_{\mathrm{s}}= \pm 1$ states. Consequently, the orientation and stability of the $\mathrm{NV}$ axis can be determined by monitoring the Zeeman splitting induced by a fixed-amplitude $(1.5 \mathrm{mT})$ external magnetic field along each of the three spatial directions ( $x$-axis shown in Fig. $3 b$ ). Figure $3 c$ shows that Zeeman splitting is maximum for a specific direction of the field (specifically, for this trapped NV, $\theta=61^{\circ}$ and $\varphi=77^{\circ}$ in polar coordinates), which corresponds to the NV spin axis.

Long-term stability was evaluated by repeating these measurements after $30 \mathrm{~min}$, and we found that the NV orientation was the same within an error range of $\pm 5^{\circ}$. This demonstrates that the nanocrystal diamond has not substantially rotated within the trap. We attribute this behaviour, which was observed for the 

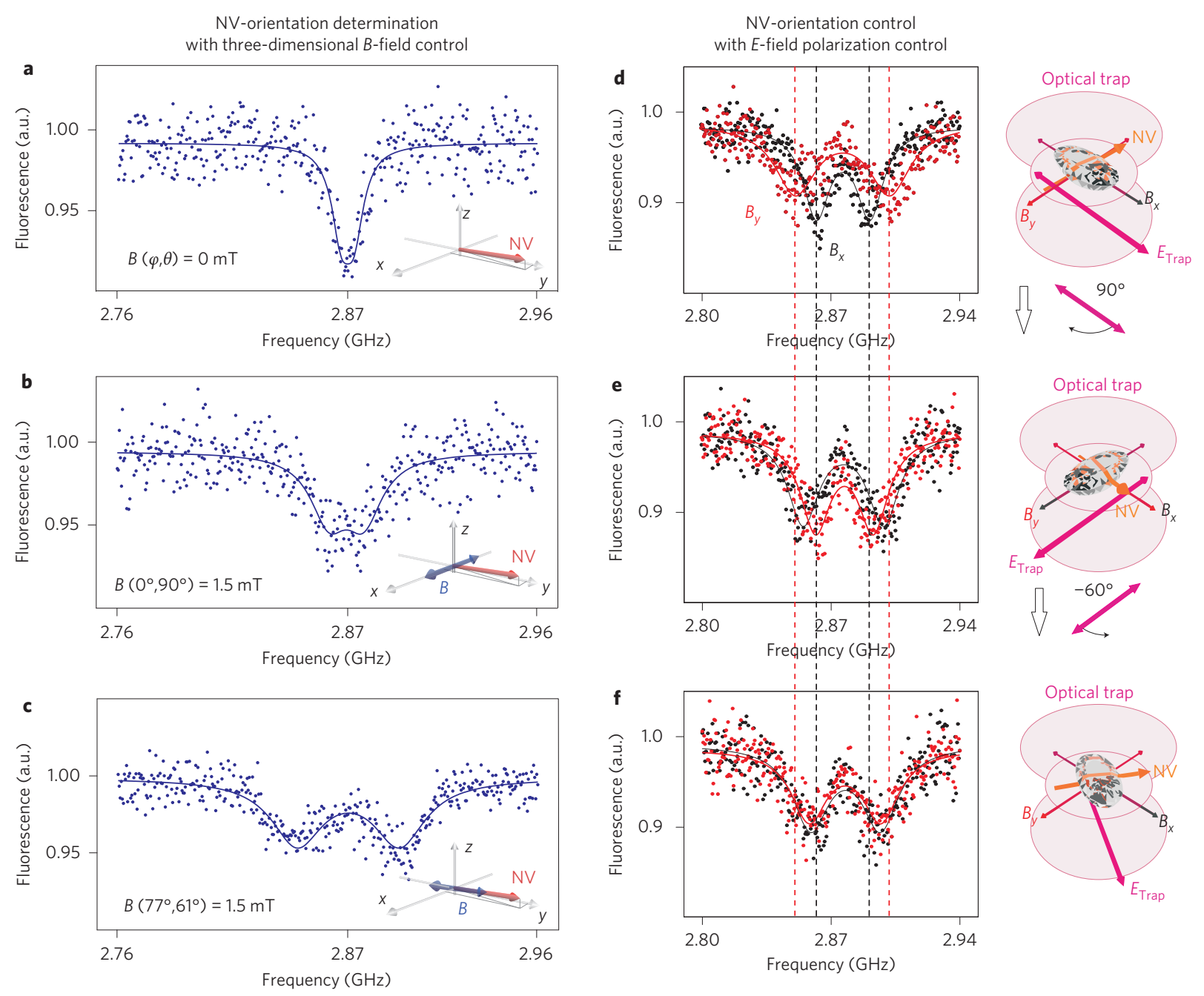

Figure 3 | Vectorial magnetometry and orientation control with an optically trapped single spin. Optically detected ESR spectra of a trapped nanocrystal diamond with a single NV centre. The data points of the frequency sweep were acquired in steps of $0.5 \mathrm{MHz}$. The solid lines are two-Lorentzian fits to determine the transition frequency. Insets: orientation of the polarization axis $E$, the NV axis and the direction of the magnetic field $B$. a-c, Determination of the NV-axis orientation. In a no magnetic field is applied. The $m_{s}= \pm 1$ ground state is degenerate. For the $X$ projection of the magnetic field onto the NV axis (b) only a small Zeeman splitting is visible. When the magnetic field is aligned with the NV axis (c), a maximal splitting is recorded. This makes vector magnetometry possible. $\mathbf{d}$-f. Control of the NV-axis orientation by rotating the polarization of the trapping laser. For each orientation of the $E$-field polarization axis, two ESR spectra were acquired for two successive orientations $x$ (black dots) and $y$ (red dots) of the magnetic field (at constant magnitude). A rotation in polarization by an angle $\Delta \varphi$ rotates the NV axis by the same angle in the $x-y$ plane, as monitored by the change in $\boldsymbol{\mu B}$. Dashed lines indicate the position of the magnetic field splitting in $\mathbf{d}$ as a guide to the eye. (Note: the ground state for this $\mathrm{NV}$ was already degenerate for $B=0$.)

majority of the trapped nanocrystal diamonds (around 80\%), to the asymmetrical shape of the nanocrystals (which translates into an asymmetrical polarizability), thus creating a preferential equilibrium axis within the linearly polarized trap ${ }^{30}$ (Supplementary Figs S10 and S12). Consequently, changing the orientation of the polarization axis of the trapping laser should allow us to control the orientation of the NV axis with respect to the optical axis. This additional degree of control over the trapped NV is demonstrated in Fig. $3 \mathrm{~d}-\mathrm{f}$, in which we monitor the orientation of the $\mathrm{NV}$ axis for three different directions of the trap polarization. For each orientation of the E-field polarization axis, two ESR spectra are acquired for two successive orientations $(x$ and $y)$ of the magnetic field (at constant magnitude). An angular change $\Delta \varphi$ in the direction of the field polarization leads to a rotation of the NV axis (projected in the $x-y$ plane) by the same angle, therefore permitting us to align the NV axis orientation relative to the $B$-field lines to be probed. Although trapping with a Gaussian beam limits rotation around a single axis, further rotation control could be achieved by introducing electric fields having components along the $z$-axis either through additional trapping beams or through beam shaping with a spatial light modulator $(\mathrm{SLM})^{31}$. This novel manipulation technique is therefore an ideal platform for probing vectorial magnetic fields in three dimensions with nanoscale resolution and a magnetic sensitivity of $40 \mu \mathrm{T} \mathrm{Hz}^{-1 / 2}$ (refs 8,32) (see Supplementary sections, 'Magnetic sensitivity and Broadening of the ESR line width').

The accurate manipulation of a single NV also provides an avenue for non-invasive probing of the LDOS by exploiting its electric dipole. We demonstrate this capability by spatially mapping the NV fluorescence lifetime, which is inversely proportional to the $\operatorname{LDOS}^{33}$ (projected along the NV axis), in the proximity of a photonic system. For this purpose, a trapped single NV centre was raster-scanned across a $\mathrm{TiO}_{2}$ channel waveguide patterned on a glass substrate. At each point of the scan (scan step, $15 \mathrm{~nm}$ ), we measured the fluorescence lifetime of the NV centre (Fig. 4a). The fluorescence intensity profile features a maximum above the waveguide. This maximum is attributed predominantly to significant autofluorescence from the $\mathrm{TiO}_{2}$ (as can be seen in the EM-CCD 
a
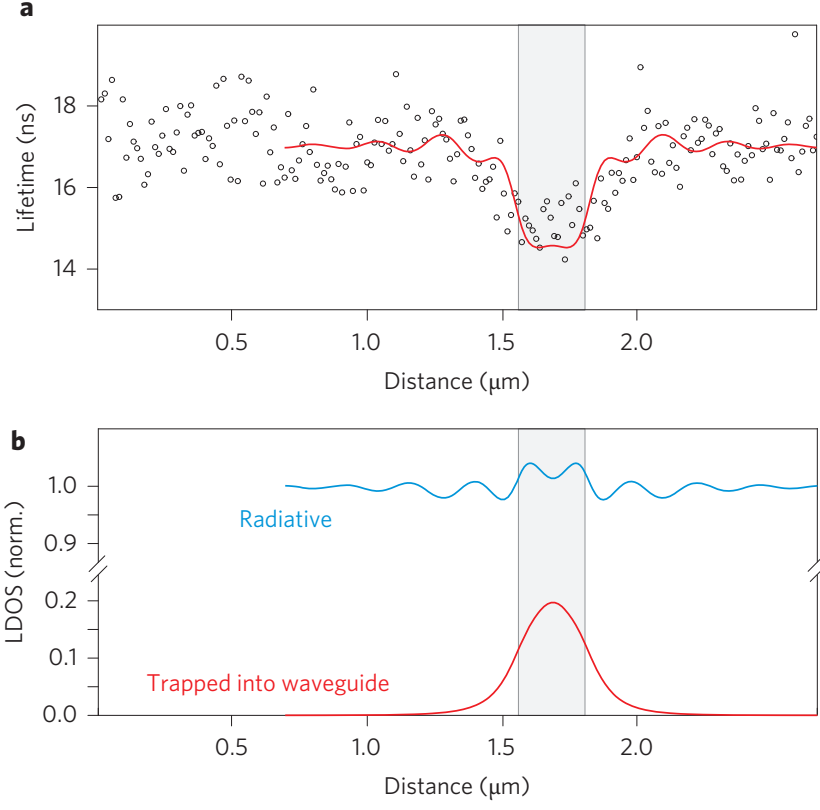

Figure 4 | LDOS mapping across a $\mathrm{TiO}_{2}$ waveguide. a, Fluorescence lifetime of the NV centre, which was acquired at different points across and over the waveguide (average over two consecutive scans). Red line: calculated

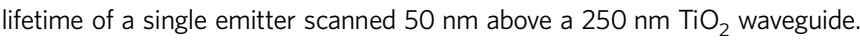
b. Calculated radiative and waveguided contributions of the LDOS for a dipole scanned $50 \mathrm{~nm}$ above a $250 \mathrm{~nm} \mathrm{TiO}{ }_{2}$ waveguide. The radiative part has only a small contribution compared with that of the non-radiative part, which is trapped in a waveguide mode and is responsible for the change in lifetime of the NV centre. The shaded area corresponds to the position of the waveguide.

fluorescence image in Fig. 1d,e). Conversely, information about the $\mathrm{NV}$-waveguide coupling and the LDOS is provided by the lifetime data, from which the contribution of the NV fluorescence can be isolated by separating its long-lived fluorescence signal from the short-lived autofluorescence of the rest of the structure using a two-exponential fit. A clear lifetime decrease is observed when the $\mathrm{NV}$ is in close proximity to the $\mathrm{TiO}_{2}$ waveguide. The lifetime is expected to be inversely proportional to the number of available photon states that are accessible to the emitting electric dipole of the NV. This quantity is also known as the LDOS, which measures the sum of field intensities of all normalized photon modes as a function of both photon energy and space position ${ }^{34}$. A decrease in lifetime of the NV centre is thus associated with an increase in the LDOS. In Fig. 4b, we show the spatial dependence of two contributions to the LDOS: radiative emission far from the system (upper curve) and non-radiative emission into a guided mode of the waveguide (lower curve). Although the former seems to depend only mildly on position, the waveguide mode contribution shows a peak that follows the observed decrease in lifetime. As shown by the red line in Fig. 4a, the theory is in very good agreement with the experiment.

Spatial and angular manipulation of optically trapped single NV centres is a simple and powerful approach that could benefit and be applied to a wide range of research. Beyond opening new opportunities for nanoscale magnetic and optical sensing in three dimensions and in liquid environments, it is non-invasive and is potentially applicable to cell biology ${ }^{35,36}$. We also foresee that an extension to multiple nanocrystal diamond trapping could be used for the implementation of flying qubits ${ }^{37}$ and nanoscale control of spin-spin interactions using plasmonic tweezer structures ${ }^{38}$, realizing a significant advance in scalable quantum computing and simulation schemes.

\section{Methods}

Optical trapping and fluorescence readout. A near-infrared $(\lambda=1064 \mathrm{~nm}) 1.5 \mathrm{~W}$ laser was split by a 50:50 beamsplitter into two beams and focused through two high-NA $(=1.2)$ water immersion objectives to a single point. The power at the objective entrance was fixed at $150 \mathrm{~mW}$ in each beam to compensate for the low transmission of the objective at this wavelength $(30 \%)$.

The nanocrystal diamonds were diluted in a 5:1 glycerol/water solution. A droplet of this solution between two coverslips served as a liquid chamber fixed on a $x y z$ piezo stage. The glycerol was chosen to increase the viscosity of the medium and consequently lower the Brownian motion of the nanocrystal diamonds. An EMCCD camera was used to detect the fluorescence of a single NV centre, and then track the single NV centres, trap them and position or scan them at desired locations in the chamber. A $532 \mathrm{~nm}$ laser was used to excite the NV centre. The emitted fluorescence was detected by two APDs, after passing through a dichroic filter and a long-pass filter at $632 \mathrm{~nm}$.

Trap characterization. Using standard position measurements based on a quadrant photodetector (QPD) ${ }^{29}$, we were able to retrieve the position histogram along each of the two directions ( $x$ and $y$ ) perpendicular to the optical axis. The FWHM of the Gaussian distribution was of the order of $80 \mathrm{~nm}$ along both directions (Fig. 2). Because position detection is known to lack accuracy for determining the trap confinement in the $z$ direction, we can only provide an estimate based on the spatial confinement of the interference fringes. Assuming that the nanocrystal diamond remains localized in one fringe at the focus, we expect its confinement to be smaller than $80 \mathrm{~nm}$

Nanodiamond preparation. The nanodiamond sample (Microdiamant, MSY $0-0.1 \mu \mathrm{m}$ ) was refluxed for 6 days at $90{ }^{\circ} \mathrm{C}$ in a $9: 1$ ratio of concentrated sulphuric and nitric acids. The nanodiamonds were collected by centrifugation and ultrasonicated using a tip probe sonotrode (Branson, 101-063-346) for $1 \mathrm{~h}$ before being further refluxed under the same conditions. The nanodiamonds were then washed with deionized water and ultrasonicated in $0.1 \mathrm{M}$ sodium hydroxide for $1 \mathrm{~h}$, rinsed with water and re-ultrasonicated in $0.1 \mathrm{M}$ hydrochloric acid. The sample was rinsed and resuspended in deionized water. Finally, several centrifugation steps were used to size select the required nanodiamond particles, and dynamic light scattering (Malvern Instruments Zetasizer NS) measurements were used to determine the size distribution of the particles $(74 \pm 15 \mathrm{~nm})$

Received 13 July 2012; accepted 14 December 2012; published online 10 February 2013

\section{References}

1. Togan, E. et al. Quantum entanglement between an optical photon and a solidstate spin qubit. Nature 466, 730-734 (2010).

2. Babinec, T. M. et al. A diamond nanowire single-photon source. Nature Nanotech. 5, 195-199 (2010).

3. Fuchs, G. D., Burkard, G., Klimov, P. V. \& Awschalom, D. D. A quantum memory intrinsic to single nitrogen-vacancy centres in diamond. Nature Phys. 7 , 789-793 (2011)

4. Neumann, P. et al. Quantum register based on coupled electron spins in a roomtemperature solid. Nature Phys. 6, 249-253 (2010).

5. Degen, C. L. Scanning magnetic field microscope with a diamond single-spin sensor. Appl. Phys. Lett. 92, 243111 (2008).

6. Balasubramanian, G. et al. Nanoscale imaging magnetometry with diamond spins under ambient conditions. Nature 455, 648-651 (2008).

7. Maze, J. R. Nanoscale magnetic sensing with an individual electronic spin in diamond. Nature 455, 644-647 (2008).

8. Taylor, J. M. High-sensitivity diamond magnetometer with nanoscale resolution. Nature Phys. 4, 810-816 (2008).

9. Rondin, L. et al. Nanoscale magnetic field mapping with a single spin scanning probe magnetometer. Appl. Phys. Lett. 100, 153118 (2012).

10. Maletinsky, P. et al. A robust scanning diamond sensor for nanoscale imaging with single nitrogen-vacancy centres. Nature Nanotech. 7, 320-324 (2012).

11. Dolde, F. et al. Electric field sensing using single diamond spins. Nature Phys. 7, 459-463 (2011)

12. Dholakia, K. \& Cizmar, T. Shaping the future of manipulation. Nature Photon. 5, 335-342 (2011)

13. Meijer, J. et al. Generation of single color centers by focused nitrogen implantation. Appl. Phys. Lett. 87, 261909 (2005).

14. Rabeau, J. R. et al. Implantation of labelled single nitrogen vacancy centers in diamond using ${ }^{15}$ N. Appl. Phys. Lett. 88, 023113 (2006).

15. Aharonovich, I., Greentree, A. D. \& Prawer, S. Diamond photonics. Nature Photon. 5, 397-405 (2011)

16. Schröder, T., Schell, A. W., Kewes, G., Aichele, T. \& Benson, O. Fiber-integrated diamond-based single photon source. Nano Lett. 11, 198-202 (2010).

17. Schröder, T. et al. A nanodiamond-tapered fiber system with high single-mode coupling efficiency. Opt. Express 20, 10490-10497 (2012).

18. Kolesov, R. et al. Wave-particle duality of single surface plasmon polaritons. Nature Phys. 5, 470-474 (2009). 
19. Schell, A. W. et al. Single defect centers in diamond nanocrystals as quantum probes for plasmonic nanostructures. Opt. Express 19, 7914-7920 (2011).

20. Schietinger, S., Barth, M., Aichele, T. \& Benson, O. Plasmon-enhanced single photon emission from a nanoassembled metaldiamond hybrid structure at room temperature. Nano Lett. 9, 1694-1698 (2009).

21. Cuche, A. et al. Near-field optical microscopy with a nanodiamond-based single-photon tip. Opt. Express 17, 19969-19980 (2009).

22. Cuche, A., Mollet, O., Drezet, A. \& Huant, S. Deterministic quantum plasmonics. Nano Lett. 10, 4566-4570 (2010).

23. Huck, A., Kumar, S., Shakoor, A. \& Andersen U. L. Controlled coupling of a single nitrogen-vacancy center to a silver nanowire. Phys. Rev. Lett. 106, 096801 (2011).

24. Marty, R., Arbouet, A., Paillard, V., Girard, C. \& Colas des Francs, G. Photon antibunching in the optical near field. Phys. Rev. B 82, 081403 (2010).

25. Van der Sar, T. et al. Nanopositioning of a diamond nanocrystal containing a single nitrogen-vacancy defect center. Appl. Phys. Lett. 94, 173104 (2009)

26. Horowitz, V. R., Alemán, B. J., Christle, D. J., Cleland, A. N. \& Awschalom, D. D. Electron spin resonance of nitrogen-vacancy centers in optically trapped nanodiamonds. Proc. Natl Acad. Sci. USA 109, 13493-13497 (2012).

27. Gruber, A. et al. Scanning confocal optical microscopy and magnetic resonance on single defect centers. Science 276, 2012-2014 (1997).

28. Manson, N. B., Harrison, J. P. \& Sellars, M. J. Nitrogen-vacancy center in diamond: model of the electronic structure and associated dynamics. Phys. Rev. B 74, 104303 (2006).

29. Neuman, K. C. \& Block, S. M. Optical trapping. Rev. Sci. Instrum. 75, 2787-2809 (2004).

30. Galajda, P. \& Ormos, P. Orientation of flat particles in optical tweezers by linearly polarized light. Opt. Express 11, 446-451 (2003).

31. Novotny, L., Beversluis, M. R., Youngworth, K. S. \& Brown, T. G. Longitudinal field modes probed by single molecules. Phys. Rev. Lett. 86, 5251-5254 (2001).

32. Dreau, A. et al. Avoiding power broadening in optically detected magnetic resonance of single NV defects for enhanced dc magnetic field sensitivity. Phys. Rev. B 84, 195204 (2011).

33. Colas Des Francs, G. et al. Single molecules probe local density of modes (LDOS) around photonic nanostructures. J. Microsc. 229, 210-216 (2008).
34. García de Abajo, F. J. \& Kociak, M. Probing the photonic local density of states with electron energy loss spectroscopy. Phys. Rev. Lett. 100, 106804 (2008).

35. Mochalin, V. N., Shenderova, O., Ho, D. \& Gogotsi, Y. The properties and applications of nanodiamonds. Nature Nanotech 7, 11-23 (2012).

36. McGuinness, L. P. et al. Quantum measurement and orientation tracking of fluorescent nanodiamonds inside living cells. Nature Nanotech. 6, 358-363 (2011).

37. Divincenzo, D. P. The Physical Implementation of Quantum Computation: Scalable Quantum Computers (Wiley, 2005).

38. Juan, M. L., Righini, M. \& Quidant, R. Plasmon nano-optical tweezers. Nature Photon. 5, 349-356 (2011)

\section{Acknowledgements}

This work was partially supported by the Spanish Ministry of Sciences (grants FIS201014834 and CSD2007-046-NanoLight.es), the European Community's Seventh Framework Program under grant ERC-Plasmolight (no. 259196) and Fundació privada CELLEX. The authors thank T. Gaebel and A. Edmonds for fruitful discussions.

\section{Author contributions}

M.G., M.L.J. and R.Q. conceived the experiment. M.G. performed the experiments. J.M.S and L.J.B. provided the treated nanodiamonds. J.R. fabricated the nanostructures, J.G.A. provided theoretical support and F.K. assisted in implementing the ESR control. All authors discussed the results and commented on the manuscript. M.G., J.G.A., F.K. and R.Q. wrote the paper.

\section{Additional information}

Supplementary information is available in the online version of the paper. Reprints and permission information is available online at http://www.nature.com/reprints. Correspondence and requests for materials should be addressed to R.Q.

\section{Competing financial interests}

The authors declare no competing financial interests. 